\title{
Distortion Related to Polarization-Mode Dispersion in Analog Lightwave Systems
}

\author{
C. D. Poole and T. E. Darcie
}

\begin{abstract}
Analog transmission in single-mode fiber using chirped sources gives rise to nonlinear distortion when polarization-mode dispersion (PMD) is present. We investigate experimentally and theoretically two mechanisms for this distortion: for chirped sources, PMD in the presence of polarization-mode coupling results in second-order distortion that is proportional to the square of the modulation frequency; when polarizationdependent loss is present, an additional second-order distortion term occurs that is independent of modulation frequency. Both mechanisms give rise to distortion that is time varying due to the sensitivity of PMD to ambient temperature changes. Numerical examples indicate that these effects can limit the capacity of analog systems that use directly modulated semiconductor lasers.
\end{abstract}

\section{INTRODUCTION}

$\mathrm{T}$ HE growing application and rapid advancement of subcarrier modulation techniques in analog lightwave systems in recent years have focused attention on mechanisms that lead to nonlinear distortion in fiber transmission [1]. Among the mechanisms that have been studied include fiber chromatic dispersion [2]-[4] and nonlinear optical effects such as self-phase modulation [4] and Brillouin scattering [5].

In this paper we investigate mechanisms for nonlinear distortion in analog lightwave systems associated with polarization-mode dispersion (PMD). Polarization-mode dispersion arises in single-mode fiber when the combined effects of noncircularly symmetric internal stresses and waveguide geometry created during manufacture cause the two polarization modes of the waveguide to propagate with different group velocities [6]. In long fiber spans (e.g., $>1 \mathrm{~km}$ ), the effect of this modal dispersion is complicated by internally and externally derived perturbations on the fiber birefringence that cause the two polarization modes to couple and interfere coherently. An important consequence of this coupling is that the dispersion in a fiber becomes sensitive to changes in ambient temperature and mechanical perturbations [7], [8]. This sensitivity to the environment has been shown to lead to time-dependent fading effects in digital systems [8].

Here we identify two PMD-related mechanisms that lead to similar fading phenomena in analog systems. The

Manuscript received January 12, 1993; revised April 23, 1993.

The authors are with AT \& T Bell Laboratories, Holmdel, NJ 07733. IEEE Log Number 9211148. first mechanism arises from the interaction between PMD and laser chirp and requires that there be coupling between polarization modes. The second mechanism arises from the interaction between PMD, laser chirp, and polarization-dependent loss (PDL). The former mechanism has been investigated previously using two different models to account for the effects of polarization-mode coupling [9], [10]. This led to greatly differing predictions as to the magnitude of harmonic distortion caused by PMD because the underlying assumptions were very different. In the present work we make minimal assumptions about the microscopic fiber properties by using a phenomenological description of PMD in which the fiber is described in terms of measurable input/output characteristics [7], [11], [12]. With this approach a surprisingly simple equation (Eq. (8)) is obtained for second-order distortion caused by PMD, the essential features of which are confirmed experimentally.

\section{THEORY}

The definitions of parameters used in this paper are given in Appendix A.

We consider the simple case of a single-frequency laser source subjected to sinusoidal intensity modulation $m(t)$ $=m_{0} \cos \omega_{m} t$. For frequencies of interest in CATV applications, laser chirp can be modeled by assuming that the instantaneous optical frequency is directly proportional to the output power, $\omega(t)=\omega_{0}+\gamma m(t)$, so that the optical field emerging from the laser is given by

$$
\boldsymbol{E}_{a}(t)=\hat{\boldsymbol{\epsilon}}_{a} E_{0} \sqrt{1+m(t)} e^{i\left(\omega_{0} t+\theta(t)\right)},
$$

where $\theta(t)=\int \gamma m(t) d t=\left(\gamma m_{0} / \omega_{m}\right) \sin \omega_{m} t$ is the time-dependent phase and $\hat{\epsilon}_{a}$ is a complex unit vector specifying the polarization of the optical field.

For small modulation indices (i.e., $m_{0} \ll 1$ ), the optical spectrum, obtained by taking the Fourier transform of (1) [13], is given by

$$
E_{a}(\omega)=E_{0} \sum_{-\infty}^{\infty} A_{n} \delta\left[\omega-\omega_{0}-n \omega_{m}\right],
$$

where $\delta[x]=1$ when $x=0$ and is zero otherwise, and the coefficients $A_{n}$ are given by

$$
A_{n}=J_{n}(\beta)+\frac{m_{0}}{4} J_{n-1}(\beta)+\frac{m_{0}}{4} J_{n+1}(\beta) .
$$


In (3) $J_{n}$ are Bessel functions and $\beta=\gamma m_{0} / \omega_{m}$ is the FM index corresponding to the laser chirp.

We now consider transmission of the laser output through a fiber containing PMD and random polarization-mode coupling. In general, such a fiber can be described by a $2 \times 2$ complex transmission (Jones) matrix in which each element of the matrix is a function of the source frequency [9], [10]. The approach we take here is to model the temporal response of the fiber by expanding the transmission matrix of the fiber about the source center frequency. With this approach the optical field $\boldsymbol{E}_{b}(t)$ emerging from a fiber is related to the Fourier transform of the input field $E_{a}(\omega)$ by [7]

$$
\boldsymbol{E}_{b}(t)=c_{+} \boldsymbol{E}_{+}(t)+c_{-} \boldsymbol{E}_{-}(t),
$$

where $\boldsymbol{E}_{+}(t)$ and $\boldsymbol{E}_{-}(t)$ are given by

$$
\begin{aligned}
\boldsymbol{E}_{ \pm}(t)= & \int_{-\infty}^{\infty} E_{a}(\omega) e^{i \omega(t-\tau)} d \omega \\
& \times\left\{\hat { \boldsymbol { \epsilon } } _ { b \pm } \operatorname { e x p } \left[i \phi_{ \pm} \pm i \frac{\Delta \tau}{2}\left(\omega-\omega_{0}\right)\right.\right. \\
& \left.+i \frac{\left(\psi^{\prime \prime} \pm \Delta \tau^{\prime} / 2\right)}{2}\left(\omega-\omega_{0}\right)^{2}\right] \\
& \left. \pm \hat{\boldsymbol{\epsilon}}_{b \mp} e^{i \phi_{\mp}} \frac{i \Delta \tau}{2}\left[\hat{\boldsymbol{\epsilon}}_{a \pm}^{\prime} \cdot \hat{\boldsymbol{\epsilon}}_{a \mp}^{*}\right]\left(\omega-\omega_{0}\right)^{2}\right\}
\end{aligned}
$$

and where we have neglected fiber loss.

The expansion in (5) has been carried out to second order, where $\omega_{0}$ is the center frequency of the source, $\hat{\epsilon}_{a \pm}$ and $\hat{\epsilon}_{b \pm}$ are the input and output principal states of polarization defined in [11], $c_{ \pm}=\hat{\epsilon}_{a} \cdot \hat{\epsilon}_{a \pm}^{*}$ are the projections of the input polarization onto the input principal states, $\psi(\omega)$ is the polarization-independent component of the output field phase, $\tau=d \psi / d \omega$ is a polarization-independent group-delay time, and $\Delta \tau$ is the differential group-delay time between the principal states. The two input (output) principal states represent the input (output) polarization states corresponding to the minimum and maximum group-delay times through the fiber. The second derivative of the phase $\psi^{\prime \prime}=d^{2} \psi / d \omega^{2}$ is the total chromatic dispersion of the fiber and can be expressed in terms of the conventional dispersion parameter $D(\mathrm{ps} / \mathrm{nm}$ $\cdot \mathrm{km})$ and the fiber length $L(\mathrm{~km}), \psi^{\prime \prime}=-\lambda^{2} D L / 2 \pi c$. The primes in (5) indicate differentiation with respect to frequency and all parameters are evaluated at the source center frequency $\omega_{0}$.

To incorporate the effects of polarization-sensitive elements in the system, such as polarization-dependent couplers, splices or receivers, we introduce polarizationdependent loss (PDL) in the form of a single partial polarizer placed at the fiber output. The partial polarizer has the property that light polarized along its pass axis sees unity power transmission $T_{\|}=1$, while light polarized orthogonal to this axis sees a slightly reduced transmission $T_{\perp}=1-\Delta T$. We define $\Delta T$ to be the polarization-dependent loss.
With the polarizer pass axis denoted by the unit vector $\hat{\epsilon}_{p}$, the optical power detected by a square-law detector positioned after the partial polarizer is given by

$$
P_{\text {out }}(t)=\left|\boldsymbol{E}_{b}(t)\right|^{2}-\Delta T\left(1-\left|\hat{\boldsymbol{\epsilon}}_{p} \cdot \boldsymbol{E}_{b}^{*}(t)\right|^{2}\right) .
$$

By allowing $\hat{\epsilon}_{p}$ to be complex, we can model any partial polarizer, even those whose pass axis corresponds to an elliptical polarization state.

The RF signal received by an ideal analog receiver is proportional to the power waveform $P_{\text {out }}(t)$. For the single-channel case considered here, the second-order nonlinear distortion in the received RF signal is given by the ratio of the RF power contained in the second harmonic of the modulation (carrier) frequency to the RF power in the carrier

$$
\eta^{(2)}=\frac{\text { RF power at } 2 \omega_{m}}{\text { RF power at } \omega_{m}}=\frac{\left|P_{\text {out }}^{(2)}\right|^{2}}{m_{0}^{2} E_{0}^{4}},
$$

where $P_{\text {out }}^{(2)}$ is the amplitude of the second-harmonic component of the optical power waveform in (6).

The ratio $\eta^{(2)}$ is obtained analytically by inserting (2) into (5) while making use of (3), (4), and (6) and the assumption of small distortions (Appendix B). The mathematics is cumbersome and the result, when represented with the parameters of (5), complicated. However, the result can be cast into a surprisingly simple and more meaningful form by representing both the dispersion and the state of polarization of the light using the Poincare sphere representation [14]. The interested reader can refer to Appendix B for the derivation. Here we simply present the result

$$
\begin{aligned}
\eta^{(2)}= & \gamma^{2} m_{0}^{2}\left\{\omega_{m}^{2}\left[\psi^{\prime \prime}-\frac{\hat{\boldsymbol{s}} \cdot \boldsymbol{\Omega}^{\prime}}{2}\right]^{2}\right. \\
& \left.+\frac{\Delta T^{2}}{16}[\hat{\boldsymbol{p}} \cdot(\boldsymbol{\Omega} \times \hat{\boldsymbol{s}})]^{2}\right\} .
\end{aligned}
$$

In (8) $\hat{\boldsymbol{s}}$ and $\hat{\boldsymbol{p}}$ are real, three-component unit vectors representing the output polarization and polarizer axis, respectively, $\boldsymbol{\Omega}$ is the output polarization-dispersion vector, and $\boldsymbol{\Omega}^{\prime}=d \mathbf{\Omega} / d \omega$ is its frequency derivative. All parameters appearing in (8) are real and, as with the parameters in (5), are evaluated at the source center frequency $\omega_{0}$. In obtaining (8) only the first nonzero terms in the frequency expansion have been retained.

The polarization-dispersion vector $\mathbf{\Omega}$ is a compact way of representing PMD in a fiber [12], [15]-[18]. The direction of $\boldsymbol{\Omega}$ identifies the output principal state of polarization on the Poincaré sphere corresponding to the minimum propagation delay time through the fiber. The magnitude of $\boldsymbol{\Omega}$ is equal to the differential group-delay time between the fast and slow principal states, i.e., $|\boldsymbol{\Omega}|=$ $\Delta \tau$. Under static conditions, PMD in a fiber is completely characterized by the vector $\boldsymbol{\Omega}$ and its frequency dependence. When the source spectrum is narrow and the effects of PMD are small, as assumed here, it is sufficient 
to know $\boldsymbol{\Omega}$ and its derivative. In the language of the frequency expansion of (5), $\boldsymbol{\Omega}$ evaluated at the source center frequency $\omega_{0}$ represents the first-order effect of PMD, while its derivative $\boldsymbol{\Omega}^{\prime}$ represents a second-order effect.

The first bracketed term inside the curly braces in (8) is the second-harmonic distortion arising from the interaction of fiber dispersion and laser chirp. It contains the contribution from chromatic dispersion ( $\psi^{\prime \prime}$ term) and from PMD (the vector dot product). The second bracketed term is the distortion arising from the interaction of PMD, laser-chirp, and polarization-dependent loss.

The origin of the second-harmonic distortion terms in (8) can be understood qualitatively as arising from the beating of the carrier with low-level amplitude-modulated signals that are created by frequency-to-amplitude modulation conversion in the system. In the case of the pure dispersion terms in (8), the frequency modulation of the laser is converted to amplitude modulation by the frequency-dependent group-delay time (i.e., dispersion) in the fiber, which causes the optical power to be redistributed in time at the fiber output. In the case of the polarization-dependent loss mechanism, the frequency modulation is first converted to polarization modulation by the PMD of the fiber. This polarization modulation is then converted to amplitude modulation when the signal light passes through an element whose loss (or transmission) is polarization-dependent.

Several important conclusions can be drawn from (8). First, the equation shows that in the absence of polarization-dependent loss (i.e., $\Delta T=0$ ), PMD-induced distortion arises from the frequency dependence of the dispersion vector, represented here by its derivative $\boldsymbol{\Omega}^{\prime}$. There is no term involving $\boldsymbol{\Omega}$ itself. This is because a differential group-delay time $\Delta \tau=|\boldsymbol{\Omega}|$ between orthogonal components of the optical field does not in itself give rise to distortion. Furthermore, since the frequency dependence of the dispersion vector becomes large only when there is significant polarization-mode coupling, it can be inferred that polarization-mode coupling is necessary for PMD-induced distortion, a conclusion that has been arrived at elsewhere [9], [10]. Thus, for example, we would expect little distortion when transmitting through a polarizationmaintaining fiber, regardless of the launch polarization or level of PMD.

A second point to note is that the pure dispersion terms scale with the modulation frequency squared, while the polarization-dependent loss term is independent of modulation frequency. Therefore, high-frequency channels are more likely to be affected by the pure dispersion mechanism, while low-frequency channels are more likely to be affected by the polarization-dependent loss mechanism.

A third point to note is that there are special conditions in which each of the PMD distortion terms are identically zero, regardless of the amount of PMD. For example, the pure PMD distortion term goes to zero when the output polarization is orthogonal to the derivative of the dispersion vector (i.e., when $\hat{s} \cdot \Omega^{\prime}=0$ ). The polarization-de- pendent loss term, on the other hand, will be zero when the polarization is parallel to the dispersion vector (i.e., when $\boldsymbol{\Omega} \times \hat{\boldsymbol{s}}=0$ ) or when this cross product is orthogonal to the polarizer axis (i.e., $\hat{p} \cdot(\boldsymbol{\Omega} \times \hat{s})=0)$. In general, there is no single polarization state that will eliminate both terms at the same time. However, when one term can be neglected, for example, when there is negligible polarization-dependent loss, adjustment of the launch polarization can be used to eliminate the distortion.

In general, PMD-related distortion is determined by the relative angles of the vectors in (8), as well as the magnitudes of $\boldsymbol{\Omega}$ and $\boldsymbol{\Omega}^{\prime}$. Since these parameters depend on the details of the birefringence along the fiber path and since this birefringence is random, the distortion cannot be known a priori. Furthermore, the distortion will vary randomly in a single fiber through variations in ambient temperature and changes in the perturbations acting on the fiber.

To quantify the magnitude of the affect under these conditions, it is useful to consider the ensemble average distortion that would arise among a population of statistically equivalent fibers. This average is obtained by taking the average of (8), assuming a uniform distribution of the polarization vector $\hat{s}$ over the Poincaré sphere, and is given by

$$
\begin{aligned}
\left\langle\eta^{(2)}\right\rangle= & \gamma^{2} m_{0}^{2}\left\{\omega_{m}^{2}\left[\psi^{\prime \prime 2}+\frac{\left\langle\left|\mathbf{\Omega}^{\prime}\right|^{2}\right\rangle}{12}\right]\right. \\
& \left.+\frac{\Delta T^{2}\left\langle|\mathbf{\Omega}|^{2}\right\rangle}{72}\right\} .
\end{aligned}
$$

In obtaining (9) we have made use of the relations $\left\langle\cos ^{2} \theta\right\rangle=1 / 3$ and $\left\langle\sin ^{2} \theta\right\rangle=2 / 3$, where $\theta$ is the angle between 3-dimensional vectors. Under reasonable assumptions it is expected that (9) can be generalized to also represent the average obtained in a single fiber subjected to varying ambient temperature [8], [19].

Equation (9) can be simplified further by assuming that the fiber is long compared to the characteristic coupling length of the modes, $L \gg l_{c}$. For spans many kilometers long this is likely to be a good approximation since the longest reported coupling lengths have been less than a kilometer [20]. Using this assumption the average magnitude of the dispersion vector $\Omega$ is simply related to the average magnitude of its derivative $\Omega^{\prime}$ by [16]

$$
\begin{aligned}
\left\langle\left|\boldsymbol{\Omega}^{\prime}\right|^{2}\right\rangle & =\frac{\left\langle|\boldsymbol{\Omega}|^{2}\right\rangle^{2}}{3} \\
& =\frac{\left\langle\Delta \tau^{2}\right\rangle^{2}}{3} \quad\left(L / l_{c} \gg 1\right) .
\end{aligned}
$$

Making use of the relation $\left\langle\Delta \tau^{2}\right\rangle=(3 \pi / 8)\langle\Delta \tau\rangle^{2}$ corresponding to a Maxwell distribution for $\Delta \tau$ at long lengths 
[12], [16], [21], (9) takes the form

$$
\begin{aligned}
\left\langle\eta^{(2)}\right\rangle= & \gamma^{2} m_{0}^{2}\left\{\psi^{\prime \prime 2} \omega_{m}^{2}+\frac{\pi^{2} \omega_{m}^{2}\langle\Delta \tau\rangle^{4}}{256}\right. \\
& \left.\left.+\frac{\pi \Delta T^{2}\langle\Delta \tau\rangle^{2}}{192}\right\} \quad \text { (single channel, } L / l_{c} \gg 1\right) .
\end{aligned}
$$

\section{A. Multiple-Channel Case}

So far we have considered the case of a single-channel system. In multiple-channel systems many carriers are transmitted simultaneously. The resulting nonlinear distortion arises through mixing of the carriers to create sum and difference frequency components corresponding to the various permutations of the channel frequencies. This is manifested in the RF frequency spectrum by a collection of distortion peaks that fall near the carrier peaks. The RF power in the largest of the peaks falling near a given carrier is used as a measure of the composite second-order (CSO) distortion for that channel. Because the channels are typically spaced in a regular pattern, many sum and difference frequency signals fall at the same place in the RF spectrum. As a result most CSO peaks consist of more than one signal.

To generalize the single-channel result given in (11) to the multichannel case, a parameter $N$ (referred to as the product count) is introduced to represent the number of channel pairs contributing to a given CSO peak. This number, which depends on the particular channel and the frequency allocation in a given system, can be calculated once the channel frequencies are known.

In addition to the product count, the multichannel system differs from the single-channel case because most of the CSO peaks are produced by the mixing of two different channels. This nondegenerate mixing produces second-order distortion that is a factor of 4 larger in RF power than the second-harmonic distortion of a single channel (see Appendix C). Using this factor of 4 together with the product count $N$ and (11), we obtain the CSO in a multichannel system

$$
\begin{aligned}
\langle\mathrm{CSO}\rangle= & N \gamma^{2} m_{0}^{2}\left\{\psi^{\prime \prime 2} \omega_{d}^{2}+\frac{\pi^{2} \omega_{d}^{2}\langle\Delta \tau\rangle^{4}}{256}\right. \\
& \left.+\frac{\pi \Delta T^{2}\langle\Delta \tau\rangle^{2}}{48}\right\} \\
& \text {-(multiple channel, } L / l_{c} \gg 1 \text { ). }
\end{aligned}
$$

where $\omega_{d}$ is now the RF frequency at which the distortion is observed. Note that in the single-channel case (11), $\omega_{d}=2 \omega_{m}$.

\section{EXPERIMENT}

Figure 1 shows the experimental multichannel CATV system used to investigate the nonlinear distortion described by (12). A CW carrier source was used to drive a

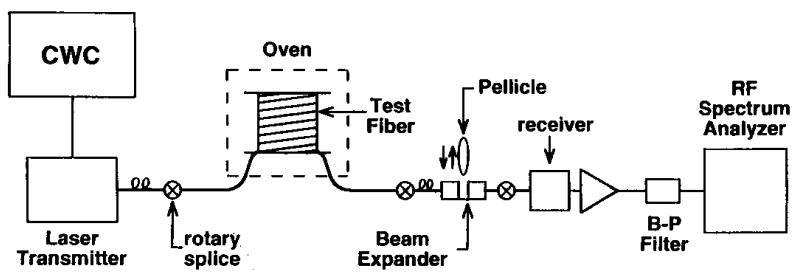
Fig. 1. Experimental CATV system. CWC is 60-channel CW carrier
source.

1.3- $\mu \mathrm{m}$ DFB laser with 60 simultaneous AM channels. The output of the transmitter was sent through the test fibers which were temperature tuned so that the sensitivity of the distortion to fiber temperature changes could be investigated. This was typically accomplished by placing the test fiber in an oven. At the output of the test fiber, a fiber-optic beam expander was placed in the optical path so that a pellicle could be inserted into the beam. The pellicle was used to introduce variable polarization-dependent loss by adjusting the angle of incidence of the light falling onto the pellicle surface. After the beam expander, the optical signal was detected by a pin receiver and the composite second-order distortion (CSO) determined by measuring the appropriate peak heights on an RF spectrum analyzer.

The PMD of each test fiber was characterized by its expected value $\langle\Delta \tau\rangle$. These values were obtained by a broadband measurement technique employing an LED source and fixed analyzer [22]. Measurements were made under static temperature conditions and the average PMD that was obtained corresponded to an average over wavelength.

In order to isolate PMD-related distortions from other distortions in our system, a DFB laser with unusually high chirp $\left(\gamma m_{0} / 2 \pi=700 \mathrm{MHz}\right)$ and fibers with unusually high PMD were used in the experiment. Because of the operating wavelength in the experiment and the relatively short fiber lengths used $(<16 \mathrm{~km})$, no effects due to chromatic dispersion were observed.

\section{A. Pure PMD Mechanism}

Initial measurements were made without polarizationdependent loss in the system. Figure 2 shows the variation of the nonlinear distortion caused by the sensitivity of the PMD to temperature. The figure shows the CSO measured through 26 ps of PMD over a 14-min period during which the temperature in the oven was being ramped upward from approximately 25 to $50^{\circ} \mathrm{C}$. Swings in the CSO through the test fiber approach $20 \mathrm{~dB}$. The CSO measured through a short fiber jumper, shown as the baseline in Fig. 2, shows negligible variation. Measurements made through a short length of polarization-maintaining (PM) fiber with total PMD of about 30 ps showed no measurable distortion. The lack of distortion we attribute to the lack of polarization-mode coupling in the PM fiber as already discussed. All of the test fibers (other than the 
PM fiber) showed behavior qualitatively the same as that shown in Fig. 2.

In agreement with (8), the distortion could always be eliminated by adjustment of the polarization using a polarization controller at the input end of the test fiber. However, to maintain zero distortion, the controller had to be continuously adjusted in order to track changes in the fiber.

Swings in the composite third-order distortion, or composite triple beat (CTB), were also observed. The maximum levels of CTB were at least $20 \mathrm{~dB}$ less than the average CSO levels, even with the unusually high laser chirp and fiber PMD used in the measurements. Typically, the CTB was below the noise floor of the measurement. We do not anticipate that CTB variations will be measurable for typical component values.

To compare theory to experiment, the average composite second-order (CSO) distortion was measured by taking a time average of CSO data similar to that shown in Fig. 2. Typically 30 -min data scans were used during which time the ambient temperature of the fiber was being ramped from 25 to $50^{\circ} \mathrm{C}$.

Figure 3 shows the average CSO measured as a function of PMD without polarization-dependent loss. The PMD was varied by using different combinations of test fibers. Data for four different AM channels are shown. The estimated error in the PMD values is indicated by the error bar on the bottommost data point. The data clearly indicate a strong dependence on the PMD. The ordering of the channels for a given PMD value is indicative of a strong modulation-frequency dependence in agreement with (12). The two lowest channel 60 data points (triangles) were obtained using a linearization circuit on the transmitter to cancel the inherent transmitter distortion and allow measurements at these low distortion values [23].

The curves shown in Fig. 3 were generated using the second term in (12) together with independently measured modulation parameters $\gamma$ and $m_{0}$ and the known composite product counts. The figure shows reasonable agreement between the experimental data points and the theoretical curves.

\section{B. Polarization-Dependent Loss Mechanism}

Distortion caused by the presence of polarization-dependent loss was investigated by inserting the pellicle into the beam expander. Since, according to (12), low PMD and low modulation frequency favor the polarization-dependent loss mechanism over the pure PMD mechanism, polarization-dependent loss effects were isolated by using one of the lower PMD fibers and a low-frequency channel (channel 3,61.25 MHz) so that polarization-dependent loss would be the dominant mechanism.

Figure 4 shows the average CSO as a function of the polarization-dependent loss $\Delta T$, introduced by positioning the pellicle at different angles with respect to the beam. The polarization-dependent loss was measured directly by adjusting the polarization incident on the pellicle using a

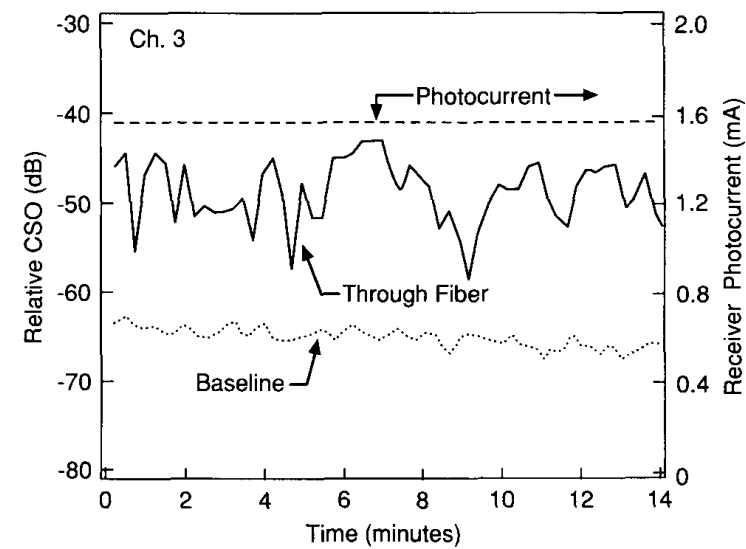

Fig. 2. Time-dependent fluctuation of the CSO caused by varying the fiber temperature.

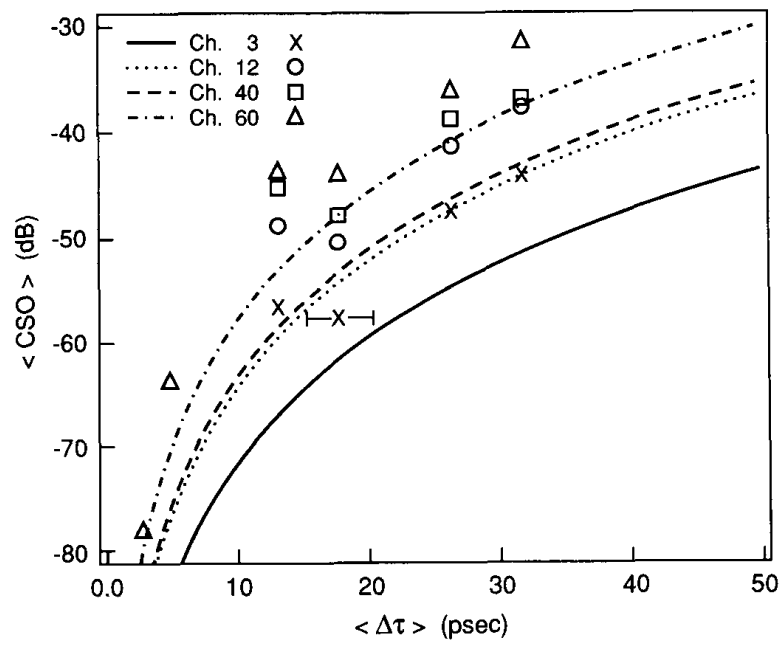

Fig. 3. Average CSO versus PMD $=\langle\Delta \tau\rangle$ (without polarization-dependent loss) for four different channels. Curves correspond to theory. Laser chirp, $\gamma m_{0} / 2 \pi=700 \mathrm{MHz}$. Channel frequencies are $f_{3}=61.25$ $\mathrm{MHz}, f_{12}=205.25 \mathrm{MHz}, f_{40}=319.25 \mathrm{MHz}, f_{60}=433.25 \mathrm{MHz}$. Product counts are $N=48,25,12,22$, respectively.

polarization controller to find the minimum and maximum transmission. As in Fig. 3, each data point in Fig. 4 is an average taken over a 30 -min time span while the oven temperature was being cycled. The theoretical curve shown in the figure was generated from the third term in (12).

\section{Discussion}

The importance of the mechanisms described here for practical analog systems is illustrated in Fig. 5. The figure shows the system parameter requirements for a standard U.S. 60-channel CATV system to operate with an average composite second-order distortion of $-70 \mathrm{dBc}$. The average CSO value of $-70 \mathrm{dBc}$ was chosen to provide a safety margin for fluctuations in the CSO before a distortion level of $-55 \mathrm{dBc}$ is reached. Above this level of distortion noticeable degradation of the video picture can occur. 


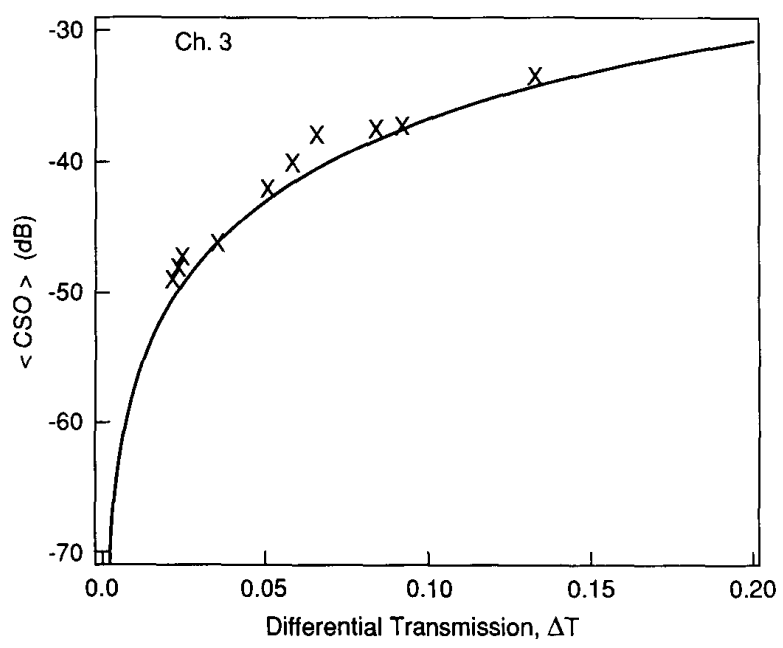

Fig. 4. Average CSO versus polarization-dependent loss for channel 3. Curves correspond to theory. $\langle\Delta \tau\rangle=18 \mathrm{ps}$.

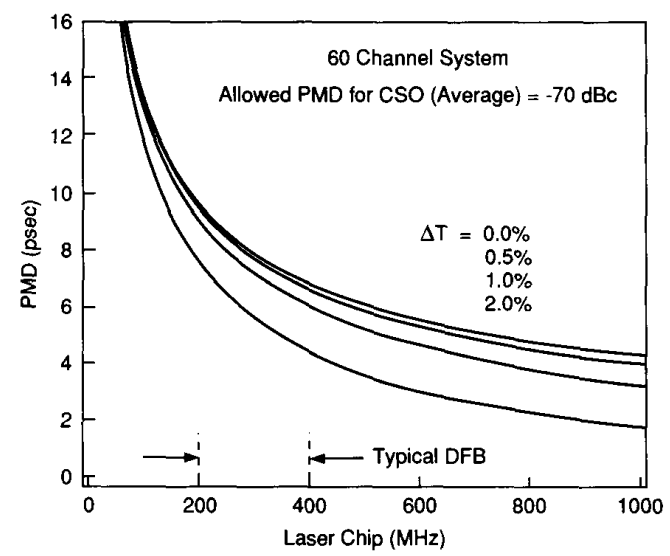

Fig. 5. Required system parameters for a 60 -channel CATV system to achieve an average CSO of $-70 \mathrm{dBc}$. PMD $=\langle\Delta \tau\rangle$. Laser chirp $=$ $\gamma m_{0} / 2 \pi$ in $\mathrm{MHz}$. $\Delta T=$ polarization-dependent loss. Product count, $N=22$.

The two most important parameters, fiber PMD and laser chirp, are indicated on the vertical and horizontal axes, respectively. The four curves shown in the figure correspond to different levels of polarization-dependent loss. Each curve was generated using (12) assuming a modulation frequency of $433.25 \mathrm{MHz}$, corresponding to the highest frequency channel in a standard 60-channel system. Also shown in the figure is the typical range for laser chirp.

Figure 5 shows that a 60 -channel system should have an average span PMD below about 7 ps in the absence of polarization-dependent loss and below about 4 ps with $2 \%$ polarization-dependent loss.
Although Fig. 5 shows that polarization-dependent loss as low as $2 \%$ can have a significant effect on the PMD requirements for analog systems, it has been assumed that the polarization-sensitive element is located at the output end of the fiber span, which is the worst-case situation. Polarization-sensitive elements at the input end will not cause distortion, and elements distributed along the length of a span will have less effect than if positioned at the end. However, our use of a single partial polarizer located at the output of the fiber is directly relevant to the use of optical couplers at the output end of systems for powersplitting or WDM applications. Such couplers often have polarization-dependent transmission of the order of $10 \%$. Stringent requirements on the polarization dependence of such couplers and/or fiber PMD will be required to avoid significant distortion.

Finally, it should be noted that while our central result, (8), indicates that there is no distortion when the source chirp is zero, this is true only to within the accuracy of the frequency expansions that were used to obtain the two terms in (8). Since the terms in (8) represent a very small effect, it is expected that the next higher-order terms in the expansions will be far smaller. Thus, the use of chirpfree sources, for example, those using external modulators, should greatly reduce the effects described here.

\section{CONCLUSION}

We have investigated experimentally and theoretically two mechanisms for nonlinear distortion in analog lightwave systems associated with PMD. The first mechanism requires polarization-mode coupling and laser chirp and creates a distortion (measured in RF power) that scales as the fourth power of PMD and as the square of both the modulation frequency and the laser chirp. The second mechanism results from an interaction between PMD and polarization-dependent loss and creates a distortion that scales as the square of the PMD, the laser chirp, and the polarization-dependent loss but is independent of modulation frequency. Both mechanisms require laser chirp and create distortion that is sensitive to changes in fiber temperature and mechanical perturbations.

\section{APPENDIX A}

\section{Parameter Definitions}

$\begin{array}{ll}\omega_{m} & \begin{array}{l}\text { Modulation frequency (radian) of laser output } \\ \omega_{d}\end{array} \\ m_{0} & \begin{array}{l}\text { Distortion frequency (radian) } \\ \text { Amplitude of sinusoidal intensity modulation } \\ \text { emerging from laser }\end{array} \\ \omega & \begin{array}{l}\text { Optical frequency (radian) of laser output } \\ \omega_{0}\end{array} \\ \gamma & \begin{array}{l}\text { Optical frequency of unmodulated laser output } \\ \text { Chirp parameter that relates change in laser }\end{array} \\ \beta & \begin{array}{l}\text { output frequency to change in laser output power } \\ \beta=\gamma m_{0} / \omega_{m}\end{array} \\ E_{0} & \begin{array}{l}\text { Electric field amplitude of unmodulated laser } \\ \text { output }\end{array}\end{array}$


$E_{a, b} \quad$ Electric field amplitudes of optical signal at input $\left(E_{a}\right)$ and output $\left(E_{b}\right)$ of fiber

$A_{n} \quad$ Bessel function coefficients

$\delta[x] \quad$ Dirac delta function

$J_{n}(x) \quad$ Bessel function

$\hat{\epsilon}_{a \pm} \quad$ Complex unit vectors specifying input principal states of polarization

$\hat{\epsilon}_{b \pm} \quad$ Complex unit vectors specifying output principal states of polarization

$\Delta \tau \quad$ Difference in arrival time for waves launched with polarizations aligned with the two input principal states of polarization

$L \quad$ Fiber length

$D \quad$ Length-normalized chromatic dispersion typically represented in the units $\mathrm{ps} / \mathrm{nm} \cdot \mathrm{km}$

$\psi^{\prime \prime} \quad$ Total chromatic dispersion of fiber, $\psi^{\prime \prime}=$ $-\lambda^{2} D L / 2 \pi$

$\Delta T \quad$ Polarization-dependent loss defined as the difference between maximum and minimum transmission, $\Delta T \leq 1$

$\hat{\epsilon}_{p} \quad$ Complex unit vector specifying pass axis of general partial polarizer

$P_{\text {out }}^{(2)} \quad$ Amplitude of the second-harmonic component of output optical power waveform

$\eta^{(2)} \quad$ Ratio of RF power at second harmonic to RF power at carrier frequency

$\boldsymbol{\Omega} \quad$ Three-dimensional polarization-dispersion vector (Poincaré sphere representation), where $|\boldsymbol{\Omega}|$ $=\Delta \tau$

$\hat{s} \quad$ Real unit vector (Poincaré sphere representation) specifying polarization of unmodulated optical signal at fiber output

$\hat{p} \quad$ Real unit vector (Poincaré sphere representation) specifying pass axis of general partial polarizer

$l$. Coupling length corresponding to distance over which power is coupled between polarization modes

$N \quad$ Product count, equal to the number of mixing products contributing to a distortion peak in the RF spectrum of a multichannel system

$\langle\mathrm{CSO}\rangle$ Average composite second-order distortion

$\mathrm{dBc} \quad$ Unit of RF power, equal to $10^{*} \log$ of the ratio of the RF power to the RF power in the carrier

\section{APPENDIX B}

In this appendix we derive the second-harmonic signal ((8) of the text) created by transmitting a single-channel analog signal through a fiber containing PMD, chromatic dispersion, and polarization-dependent loss. The contribution from dispersion alone and the contribution from polarization-dependent loss are treated separately.

\section{Pure Dispersion Mechanism}

Here we derive the first bracketed term in (8). This term is due to fiber chromatic and polarization-mode dispersion interacting with laser chirp.
We assume that the optical spectrum of the source is sufficiently narrow that the exponential in (5) can be expanded, so that the equation takes the form

$$
\begin{aligned}
\boldsymbol{E}_{ \pm}(t)= & \int_{-\infty}^{\infty} E_{a}(\omega) e^{i \omega(t-\tau)} d \omega \\
& \times\left\{\hat { \epsilon } _ { b \pm } e ^ { i \phi _ { \pm } } \left[1 \pm i \frac{\Delta \tau}{2}\left(\omega-\omega_{0}\right)-\frac{\Delta \tau^{2}}{8}\left(\omega-\omega_{0}\right)^{2}\right.\right. \\
& \left.+i \frac{\left(\psi^{\prime \prime} \pm \Delta \tau^{\prime} / 2\right)}{2}\left(\omega-\omega_{0}\right)^{2}\right] \\
& \left. \pm \hat{\epsilon}_{b \mp} e^{i \phi_{\mp}} \frac{i \Delta \tau}{2}\left[\hat{\epsilon}_{a \pm}^{\prime} \cdot \hat{\epsilon}_{a \mp}^{*}\right]\left(\omega-\omega_{0}\right)^{2}\right\}
\end{aligned}
$$

Putting (B1) into (4) of the text and squaring gives the time-dependent optical power emerging from the fiber

$$
\begin{aligned}
P_{\mathrm{out}}(t)= & E_{b}(t) \cdot E_{b}^{*}(t) \\
= & \int_{-\infty}^{\infty} \int_{-\infty}^{\infty} E_{a}(\omega) E_{a}^{*}\left(\omega^{\prime}\right) e^{i\left(\omega-\omega^{\prime}\right)(t-\tau)} d \omega d \omega^{\prime} \\
& \times\left\{1+\frac{i \Delta \tau}{2}\left(\left|c_{+}\right|^{2}-\left|c_{-}\right|^{2}\right)\left(\omega-\omega^{\prime}\right)\right. \\
& -\frac{\Delta \tau^{2}}{8}\left(\omega-\omega^{\prime}\right)^{2} \\
& +i\left[\frac{\psi^{\prime \prime}}{2}+\left(\left|c_{+}\right|^{2}-\left|c_{-}\right|^{2}\right) \frac{\Delta \tau^{\prime}}{4}+\Delta \tau a\right] \\
& \left.\cdot\left[\left(\omega-\omega_{0}\right)^{2}-\left(\omega^{\prime}-\omega_{0}\right)^{2}\right]\right\},
\end{aligned}
$$

where $a=\operatorname{Re}\left[c_{+} c_{-}^{*}\left(\hat{\epsilon}_{a+}^{\prime} \cdot \hat{\epsilon}_{a-}^{*}\right)\right],\left|c_{+}\right|^{2}+\left|c_{-}\right|^{2}=1$, and where terms to second order have been retained.

Substituting (2) for $E_{a}(\omega)$ in (B2) and integrating gives

$$
\begin{aligned}
P_{\text {out }}(t)= & E_{0}^{2} \sum_{m=-\infty}^{\infty} \sum_{n=-\infty}^{\infty} A_{n} A_{m} e^{i(n-m) \omega_{m}(t-\tau)} \\
& \times\left\{1+\frac{i}{2}\left(\left|c_{+}\right|^{2}-\left|c_{-}\right|^{2}\right) \Delta \tau(n-m) \omega_{m}\right. \\
& -\frac{\Delta \tau^{2}}{8}(n-m)^{2} \omega_{m}^{2} \\
& +i\left[\frac{\psi^{\prime \prime}}{2}+\left(\left|c_{+}\right|^{2}-\left|c_{-}\right|^{2}\right) \frac{\Delta \tau^{\prime}}{4}+\Delta \tau a\right] \\
& \left.+\left(n^{2}-m^{2}\right) \omega_{m}^{2}\right\} .
\end{aligned}
$$

With the change of indices $k=n-m$ and grouping terms of like frequency, (B3) can be written as

$$
\begin{aligned}
P_{\text {out }}(t)= & E_{0}^{2} \sum_{k=-\infty}^{\infty}\left\{\left[B_{k}-k^{2} B_{k} \frac{\Delta \tau^{2}}{8} \omega_{m}^{2}\right]\right. \\
& \cdot \cos \left[k \omega_{m}(t-\tau)\right] \\
& -\left[\frac{k B_{k}}{2}\left(\left|c_{+}\right|^{2}-\left|c_{-}\right|^{2}\right) \Delta \tau \omega_{m}\right. \\
& \left.\left.+\left(2 k C_{k}-k^{2} B_{k}\right) \omega_{m}^{2} G\right] \sin \left[k \omega_{m}(t-\tau)\right]\right\},
\end{aligned}
$$


where

$$
G=\frac{\psi^{\prime \prime}}{2}+\left(\left|c_{+}\right|^{2}-\left|c_{-}\right|^{2}\right) \frac{\Delta \tau^{\prime}}{4}+\Delta \tau a
$$

and the coefficients $B_{k}$ and $C_{k}$ are

$$
\begin{aligned}
& B_{k}=\sum_{n=-\infty}^{\infty} A_{n} A_{n-k}, \\
& C_{k}=\sum_{n=-\infty}^{\infty} n A_{n} A_{n-k} .
\end{aligned}
$$

The values of $B_{k}$ and $C_{k}$ are obtained by inserting (3) of the text into (B5) and using the Bessel function identities [24]

$$
\begin{aligned}
\sum_{n=-\infty}^{\infty} J_{n+l}(x) J_{n+j}(x)= & \delta[l-j], \\
\sum_{n=-\infty}^{\infty} n J_{n+l}(x) J_{n+j}(x)= & -l \delta[l-j]+\frac{x}{2}[\delta[l-j+1] \\
& +\delta[l-j-1]] .
\end{aligned}
$$

To first order in the modulation index $m_{0}$, the coefficients $B_{k}$ and $C_{k}$ are given by

$$
\begin{aligned}
B_{0} & =1, \\
B_{1} & =B_{-1}=\frac{m_{0}}{2}, \\
B_{k} & =0 \quad \text { for }|k|>1, \\
C_{0} & =\frac{m_{0} \beta}{2}, \\
C_{1} & =\frac{\beta}{2}+\frac{m_{0}}{4}, \\
C_{-1} & =\frac{\beta}{2}-\frac{m_{0}}{4}, \\
C_{2} & =C_{-2}=\frac{m_{0} \beta}{4}, \\
C_{k} & =0 \quad \text { for }|k|>2 .
\end{aligned}
$$

We are interested in the component of $P_{\text {out }}(t)$ at the second harmonic of the modulation frequency. This is given by the $k= \pm 2$ terms in (B4). Using the coefficient values given in (B7), the second-harmonic component of the output power is

$$
\begin{aligned}
P_{\text {out }}^{(2)}(t)= & -2 E_{0}^{2} \gamma m_{0}^{2} \omega_{m} \\
& \cdot\left[\frac{\psi^{\prime \prime}}{2}+\left(\left|c_{+}\right|^{2}-\left|c_{-}\right|^{2}\right) \frac{\Delta \tau^{\prime}}{4}+\Delta \tau a\right] \\
& \cdot \sin 2 \omega_{m}(t-\tau),
\end{aligned}
$$

where we have made the substitution $\beta=\gamma m_{0} / \omega_{m}$.

The first term within the brackets corresponds to the second-harmonic distortion caused by chromatic dispersion. This result agrees with a previous result obtained by a perturbation analysis on the wave equation [4]. The second derivative of the phase $\psi^{\prime \prime}$ can be expressed in the conventional units

$$
\psi^{\prime \prime}=-\frac{\lambda^{2}}{2 \pi c} D L,
$$

where $D$ is the dispersion (typically in $\mathrm{ps} / \mathrm{nm} \cdot \mathrm{km}$ ), $L$ is the fiber length, and $c$ is the speed of light.

The last two terms in (B8) are due to the fiber PMD. We now show that these two terms have a simple geometrical interpretation. To do this, we use the Poincaré sphere representation of polarization in which any polarization state can be represented by a real, three-component unit vector $\hat{s}=\left(s_{1}, s_{2}, s_{3}\right)$ [14]. The three components of the unit vector are related to the $x$-directed and $y$-directed complex components of the $E$ field by

$$
\begin{aligned}
& s_{1}=\frac{\left|E_{x}\right|^{2}-\left|E_{y}\right|^{2}}{\left|E_{x}\right|^{2}+\left|E_{y}\right|^{2}}, \\
& s_{2}=\frac{2 \operatorname{Re}\left[E_{x} E_{y}^{*}\right]}{\left|E_{x}\right|^{2}+\left|E_{y}\right|^{2}}, \\
& s_{3}=\frac{2 \operatorname{Im}\left[E_{x} E_{y}^{*}\right]}{\left|E_{x}\right|^{2}+\left|E_{y}\right|^{2}} .
\end{aligned}
$$

Using this representation, it can be shown by direct substitution that the parameter $a=\operatorname{Re}\left[c_{+} c_{-}^{*}\left(\hat{\epsilon}_{a+}^{\prime} \cdot \hat{\epsilon}_{a-}^{*}\right)\right]$ can be expressed as

$$
a=-\frac{1}{4} \hat{s}_{\text {in }} \cdot \frac{d \hat{\boldsymbol{P}}_{a-}}{d \omega},
$$

where $\hat{\boldsymbol{s}}_{\text {in }}$ and $\hat{\boldsymbol{P}}_{a \pm}$ are unit vectors representing the input polarization and the input principal states, respectively. Recalling that $c_{ \pm}=\hat{\epsilon}_{a} \cdot \hat{\epsilon}_{a \pm}^{*}$, it can also be shown that

$$
\left(\left|c_{+}\right|^{2}-\left|c_{-}\right|^{2}\right)=\hat{s}_{\text {in }} \cdot \hat{\boldsymbol{P}}_{a+}=-\hat{s}_{\text {in }} \cdot \hat{\boldsymbol{P}}_{a-},
$$

so that the two PMD terms in (B8) become

$$
\begin{aligned}
\left(\left|c_{+}\right|^{2}\right. & \left.-\left|c_{-}\right|^{2}\right) \frac{\Delta \tau^{\prime}}{4}+\Delta \tau a \\
& =-\frac{1}{4} \hat{s}_{\text {in }} \cdot\left(\Delta \tau^{\prime} \hat{\boldsymbol{P}}_{a-}+\Delta \tau \frac{d \hat{\boldsymbol{P}}_{a-}}{d \omega}\right) .
\end{aligned}
$$

It is useful to translate the vectors in (B13) to the output of the fiber. This can be done by invoking a $3 \times 3$ unitary matrix $M$ to describe the polarization properties of the fiber so that

$$
\begin{aligned}
\hat{\boldsymbol{s}}_{\mathrm{out}} & =M \hat{\boldsymbol{s}}_{\mathrm{in}}, \\
\hat{\boldsymbol{P}}_{b-} & =M \hat{\boldsymbol{P}}_{a-}, \\
\frac{d \hat{\boldsymbol{P}}_{b-}}{d \omega} & =\frac{d M}{d \omega} \hat{\boldsymbol{P}}_{a-}+M \frac{d \hat{\boldsymbol{P}}_{a-}}{d \omega}=M \frac{d \hat{\boldsymbol{P}}_{a-}}{d \omega},
\end{aligned}
$$

where $\hat{\boldsymbol{s}}_{\text {out }}$ and $\hat{\boldsymbol{P}}_{b \pm}$ are the output polarization and principal states in the Poincaré sphere representation, respectively, and where the last equation makes use of the defining property of the principal states to set $d M / d \omega \hat{P}_{a-}=0[11]$. 
Because of the unitary nature of $M$, the angles between vectors are preserved upon transmission [25], i.e.,

$$
M \boldsymbol{x} \cdot M \boldsymbol{y}=\boldsymbol{x} \cdot \boldsymbol{y}
$$

so that, using (B14) and (B15), (B13) can be written in terms of the output vectors

$$
\begin{aligned}
\left(\left|c_{+}\right|^{2}\right. & \left.-\left|c_{-}\right|^{2}\right) \frac{\Delta \tau^{\prime}}{4}+\Delta \tau a \\
& =-\frac{1}{4} \hat{\boldsymbol{s}}_{\text {out }} \cdot\left(\Delta \tau^{\prime} \hat{\boldsymbol{P}}_{b-}+\Delta \tau \frac{d \hat{\boldsymbol{P}}_{b-}}{d \omega}\right) \\
& =-\frac{1}{4} \hat{\boldsymbol{s}}_{\text {out }} \cdot \frac{d}{d \omega}\left(\Delta \tau \hat{\boldsymbol{P}}_{b-}\right) \\
& =-\frac{1}{4} \hat{\boldsymbol{s}}_{\text {out }} \cdot \boldsymbol{\Omega}^{\prime},
\end{aligned}
$$

where $\boldsymbol{\Omega}^{\prime}=d \boldsymbol{\Omega} / d \omega=d / d \omega\left(\Delta \tau \hat{\boldsymbol{P}}_{b-}\right)$ is the derivative of the output dispersion vector [18].

Equation (B8) can now be written as

$$
\begin{aligned}
P_{\text {out }}^{(2)}(t)= & -E_{0}^{2} \gamma m_{0}^{2} \omega_{m}\left[\psi^{\prime \prime}-\frac{\hat{s}_{\text {out }} \cdot \boldsymbol{\Omega}^{\prime}}{2}\right] \\
& \cdot \sin 2 \omega_{m}(t-\tau) .
\end{aligned}
$$

Thus, the PMD-related distortion terms are proportional to the dot product of the output polarization vector and the frequency derivative of the output polarization-dispersion vector.

\section{Polarization-Dependent Loss Mechanism}

Here we derive the second-harmonic distortion caused by the interaction of PMD, laser chirp, and polarizationdependent loss. This term appears as the last term in (8) of the text and can be derived in a manner analogous to the derivation given in the previous section for the pure PMD terms by using the temporal response function given in (5) together with the second term in (6). However, this effect is most easily derived by a Poincaré sphere analysis, which has the added benefit of being more intuitive. This is the approach given here.

We start by considering the optical power transmitted through a general partial polarizer located at the output of a fiber. We let the polarizer be described by an arbitrary unit vector $\hat{p}$ (Poincaré sphere representation) and differential transmission $\Delta T$. Light having a state of polarization aligned with the polarizer axis sees unity transmission, while light polarized orthogonal to the axis sees a reduced transmission $T=1-\Delta T$. More generally, for an arbitrary incident polarization $\hat{s}$, the transmission through the partial polarizer is given by

$$
T=1-\frac{\Delta T}{2}[1-\hat{p} \cdot \hat{s}] .
$$

Note that orthogonal polarizations are represented on the Poincaré sphere by oppositely directed vectors. Thus, when the incident polarization is matched to the polarizer, $\hat{p} \cdot \hat{s}=1$ and $T=1$. When the incident polarization is orthogonal (in the electric field) to the polarizer axis, then $\hat{p} \cdot \hat{s}=-1$ and $T=1-\Delta T$.

When light is transmitted through a fiber containing PMD, the polarization at the output is frequency dependent. This effect is the frequency-domain manifestation of PMD and is expressed by the equation

$$
\frac{d \hat{\boldsymbol{s}}}{d \omega}=\mathbf{\Omega} \times \hat{\boldsymbol{s}},
$$

where $\boldsymbol{\Omega}$ is the output dispersion vector [15]-[18]. When light is passed through a partial polarizer, the frequency dependence of the polarization is converted to a frequency-dependent transmission. For small excursions in the polarization, this frequency-dependent transmission is obtained by expanding in frequency (B18)

$$
T(\omega)=T_{0}+\frac{d T}{d \omega}\left(\omega-\omega_{0}\right)+\cdots .
$$

Using (B18) and (B19), this expansion can be written in terms of the dispersion vector $\mathbf{\Omega}$ :

$$
T(\omega)=T_{0}+\frac{\Delta T}{2}[\hat{p} \cdot(\Omega \times \hat{s})]\left(\omega-\omega_{0}\right)+\cdots .
$$

We next consider the intensity-modulated signal described by (1) of the text. Owing to chirp, the optical frequency is modulated along with the intensity, so that, after passing through a fiber with PMD, both the intensity and the polarization will be time varying. To first order, the time-dependent power transmitted through a partial polarizer positioned at the fiber output becomes, according to (B21),

$$
\begin{aligned}
P_{\text {out }}(t)= & P_{\text {in }}(t-\tau) T(\omega(t-\tau)) \\
= & E_{0}^{2}(1+m(t-\tau))\left\{T_{0}+\frac{\Delta T}{2}[\hat{\boldsymbol{p}} \cdot(\Omega \times \hat{\boldsymbol{s}})]\right. \\
& \left.\cdot\left(\omega(t-\tau)-\omega_{0}\right)\right\},
\end{aligned}
$$

where $P_{\text {in }}(t)=E_{0}^{2}(1+m(t))$.

Making the substitutions $\omega(t)=\omega_{0}+\gamma m_{0} \cos \omega_{m} t$ and $m(t)=m_{0} \cos \omega_{m} t$, (B22) becomes

$$
\begin{aligned}
P_{\text {out }}(t)= & E_{0}^{2}\left\{T_{0}+\frac{\Delta T \gamma m_{0}^{2}}{4}[\hat{\boldsymbol{p}} \cdot(\boldsymbol{\Omega} \times \hat{\boldsymbol{s}})]\right. \\
& +\left(T_{0}+\frac{\Delta T \gamma}{2}[\hat{\boldsymbol{p}} \cdot(\boldsymbol{\Omega} \times \hat{\boldsymbol{s}})]\right) m_{0} \cos \omega_{m}(t-\tau) \\
& \left.+\frac{\Delta T \gamma m_{0}^{2}}{4}[\hat{\boldsymbol{p}} \cdot(\mathbf{\Omega} \times \hat{\boldsymbol{s}})] \cos 2 \omega_{m}(t-\tau)\right\}
\end{aligned}
$$

The second-harmonic component of the output power waveform is therefore

$$
P_{\mathrm{out}}^{(2)}(t)=\frac{E_{0}^{2} \Delta T \gamma m_{0}^{2}}{4}[\hat{\boldsymbol{p}} \cdot(\mathbf{\Omega} \times \hat{\boldsymbol{s}})] \cos 2 \omega_{m}(t-\tau) .
$$


Making use of (B17) and (B24) and (7) of the text, the total second-harmonic distortion caused by fiber dispersion and polarization-dependent loss becomes

$$
\begin{aligned}
\eta^{(2)}=\frac{\left|P_{\text {out }}^{(2)}(t)\right|^{2}}{m_{0}^{2} E_{0}^{4}}= & \gamma^{2} m_{0}^{2}\left\{\omega_{m}^{2}\left[\psi^{\prime \prime}-\frac{\hat{\boldsymbol{s}} \cdot \boldsymbol{\Omega}^{\prime}}{2}\right]^{2}\right. \\
& \left.+\frac{\Delta T^{2}}{16}[\hat{\boldsymbol{p}} \cdot(\boldsymbol{\Omega} \times \hat{\boldsymbol{s}})]^{2}\right\} .
\end{aligned}
$$

\section{APPENDIX C}

In this appendix we derive the factor of 4 that was used to obtain (12) of the text from the single-channel result (11). This factor reflects the larger distortion that arises from nondegenerate mixing of carriers.

We consider a multichannel analog system consisting of $M$ CW carrier channels modulating a single semiconductor laser at channel frequencies $\omega_{j}$, where $j=$ $1,2,3, \cdots, M$. The time-varying component of the laser output power obtained by squaring (1) is given by

$$
P(t)=E_{0}^{2} m_{0} \sum_{j=1}^{M} \cos \omega_{j} t .
$$

Second-order nonlinear distortion arises in an analog system when the carriers in (C1) beat against a second signal with identical frequency content created by nonlinear mechanisms in the system. For the distortion mechanisms considered here, this second signal arises from frequency-to-amplitude modulation conversion caused by fiber dispersion and polarization-dependent loss. We represent this signal by

$$
\delta P(t)=\epsilon_{1} \sum_{j=1}^{M} \cos \omega_{j} t+\epsilon_{2} \sum_{j=1}^{M} \omega_{j} \sin \omega_{j} t,
$$

where typically $\epsilon_{1}, \epsilon_{2} \ll m_{0}$. The first term in (C2) corresponds to amplitude modulation created when a frequency-modulated signal passes through a fiber containing PMD and then through a polarization-dependent loss element (see Appendix B). The second term in (C2) corresponds to amplitude modulation created when a frequency-modulated signal passes through a dispersive fiber. This latter term can be obtained directly from (B4), by setting the modulation index $m_{0}$ to zero, while maintaining a finite chirp index $\beta$.

Second-order distortion comes from the beating of the signals in ( $\mathrm{C} 1)$ and (C2). The amplitude of this beat signal is proportional to the product of $(\mathrm{C} 1)$ and $(\mathrm{C} 2)$ :

$$
\begin{aligned}
P_{\text {beat }}(t)= & A \delta P(t) \cdot P(t) \\
= & A_{1} \sum_{j=1}^{M} \sum_{k=1}^{M} \cos \omega_{k} t \cos \omega_{j} t \\
& +A_{2} \sum_{j=1}^{M} \sum_{k=1}^{M} \omega_{j} \sin \omega_{k} t \cos \omega_{j} t .
\end{aligned}
$$

Using well-known trigonometric identities, (C3) can be rewritten as

$$
\begin{aligned}
P_{\text {beat }}(t)= & \frac{A_{1}}{2} \sum_{j=1}^{M} \sum_{k=1}^{M}\left[\cos \left(\omega_{j}+\omega_{k}\right) t+\cos \left(\omega_{j}-\omega_{k}\right) t\right] \\
& \times \frac{A_{2}}{2} \sum_{j=1}^{M} \sum_{k=1}^{M} \omega_{j}\left[\sin \left(\omega_{j}+\omega_{k}\right) t\right. \\
& \left.+\sin \left(\omega_{j}-\omega_{k}\right)\right] .
\end{aligned}
$$

The beat signals in (C4) consist of degenerate $(j=k)$ and nondegenerate $(j \neq k)$ mixing products. The degenerate mixing products are at the second harmonics of the carriers and are generated by the beating of the carriers against themselves. The nondegenerate products are at sum and difference frequencies of the carriers and are generated by the beating of two different carriers. We write these two groups of terms separately

$$
\begin{aligned}
\text { Degenerate terms }= & \frac{A_{1} M}{2}+\frac{A_{1}}{2} \sum_{j=1}^{M} \cos 2 \omega_{j} t \\
& +\frac{A_{2}}{2} \sum_{j=1}^{M} \omega_{j} \sin 2 \omega_{j} t,
\end{aligned}
$$

Nondegenerate terms

$$
\begin{aligned}
& =A_{1} \sum_{j=k+1}^{M} \sum_{k=1}^{M}\left[\cos \left(\omega_{j}+\omega_{k}\right) t\right. \\
& \left.+\cos \left(\omega_{j}-\omega_{k}\right) t\right] \\
& \quad+\frac{A_{2}}{2} \sum_{j=k+1}^{M} \sum_{k=1}^{M}\left(\omega_{j}+\omega_{k}\right) \sin \left(\omega_{k}+\omega_{j}\right) t \\
& \quad+\left(\omega_{j}-\omega_{k}\right) \sin \left(\omega_{k}-\omega_{j}\right) t .
\end{aligned}
$$

In the last equation the range of summation for the $j$ index was changed to group terms of the same frequency.

Using (C5), we compare the RF power in a nondegenerate mixing product to the $\mathrm{RF}$ power in a degenerate mixing product at the same frequency. Thus, considering the sum-frequency terms, we set $\omega_{j}+\omega_{k}=2 \omega_{j}$. The ratio of RF powers is

$$
\frac{\text { RF power sum frequency }}{\text { RF power degenerate }}=\frac{\left[A_{1}^{2}+\frac{A_{2}^{2}}{4}\left(2 \omega_{j}\right)^{2^{\prime}}\right]}{\frac{A_{1}^{2}}{4}+\frac{A_{2}^{2}}{4} \omega_{j}^{2}}=4 .
$$

A similar result can be obtained for the differencefrequency terms.

Since the vast majority of the distortion products in a multichannel system are nondegenerate products, we conclude from (C6) that the second-order distortion products in a multichannel system are a factor of 4 larger than the second-harmonic distortion products in a single channel transmitted through the same medium. 


\section{ACKNOWLEDGMENT}

The authors would like to thank A. Gnauck for providing the linearization circuit used in some of the measurements and G. E. Bodeep and A. R. McCormick for valuable technical assistance. We would also like to thank A. Alves, A. R. Chraplyvy, W. T. Greene, A. R. Judy, C. McGrath, M. R. Phillips, J. J. Refi, J. Stone, R. W. Tkach, and J. H. Winters for helpful discussions.

\section{REFERENCES}

[1] T. E. Darcie and G. E. Bodeep, "Lightwave subcarrier CATV transmission systems," IEEE Trans. Microwave Theory Technol., vol. 38 , pp. 524-533, 1990.

[2] G. J. Meslener, "Chromatic dispersion induced distortion of modulated monochromatic light employing direct detection," IEEE $J$. Quantum Electron., vol. 20, pp. 1208-1216, Oct. 1984.

[3] E. E. Bergman, C.-Y. Kuo, and S. Y. Huang, "Dispersion-induced composite second-order distortion at $1.5 \mu \mathrm{m}$," IEEE Photon. Technol. Lett., vol. 3, pp. 59-61, Jan. 1991.

[4] M. R. Phillips, T. E. Darcie, D. Marcuse, G. E. Bodeep, and N. J. Frigo, "Nonlinear distortion generated by dispersive transmission of chirped intensity-modulated signals," IEEE Photon. Technol. Lett., vol. 3, pp. 481-483, May 1991.

[5] X. P. Mao et al., "Brillouin scattering in externally modulated lightwave AM-VSB CATV transmission systems," IEEE Photon. Technol. Lett., vol. 4, pp. 287-289, 1992.

[6] I. P. Kaminow, "Polarization in optical fibers," IEEE J. Quantum Electron., vol. QE-17, pp. 15-22, 1981.

[7] C. D. Poole and C. R. Giles, "Polarization-dependent pulse compression and broadening due to polarization dispersion in dispersion-shifted fiber," Opt. Lett., vol. 13, pp. 155-157, 1988.

[8] C. D. Poole, R. W. Tkach, A. R. Chraplyvy, and D. A. Fishman, "Fading in lightwave systems due to polarization-mode dispersion," Photon Technol. Lett., vol. 3, pp. 68-70, 1991.

[9] K. Petermann, "Nonlinear transmission behavior of a single-mode fiber transmission line due to polarization coupling," in 7th European Conference on Optical Communications, ECOC' '81, paper 3.2.

[10] C. J. Nielsen, "Influence of polarization mode coupling on the transmission bandwidth of single-mode fibers," J. Opt. Soc. Amer., vol. 72 , pp. $1142-1146,1982$.

[11] C. D. Poole and R. E. Wagner, "Phenomenological approach to polarization dispersion in long single-mode fibers," Electron. Lett., vol. 22, pp. 1029-1030, 1986.

[12] C. D. Poole, J. H. Winters, and J. A. Nagel, "Dynamical equation for polarization dispersion," Opt. Lett., vol. 16, pp. 372-374, 1991.

[13] See, for example, K. S. Shanmugan, Digital and Analog Communications Systems. New York: Wiley, 1979, chap. 6.

[14] See, for example, M. Born and E. Wolf, Principles of Optics, 6th ed. New York: Pergamon, 1980, chap. 1.

[15] D. Andresciani, F. Curti, F. Matera, and B. Daino, "Measurements of group-delay difference between the principal states of polarization on a low-birefringence terrestrial cable," Opt. Lett., vol. 12, pp. 844-846, 1987.

[16] G. J. Foschini and C. D. Poole, "Statistical theory of polarization dispersion in single-mode fibers," J. Lightwave Technol., vol. 9, pp. 1439-1456, 1991 .

[17] N. S. Bergano, C. D. Poole, and R. E. Wagner, "Investigation of polarization dispersion in long lengths of single-mode fiber using multilongitudinal mode lasers," J. Lightwave Technol., vol. 5, pp. 1618-1622, 1987

[18] C. D. Poole, N. S. Bergano, R. E. Wagner, and H. J. Schulte, "Polarization dispersion and principal states in a $147 \mathrm{~km}$ undersea lightwave cable," J. Lightwave Technol., vol. 6, pp. 1185-1190, 1988.

[19] C. De Angelis, A. Galtorossa, G. Gianello, F. Matera, and M. Schiano, "Time evolution of polarization mode dispersion in long terrestrial links," J. Lightwave Technol., vol. 10, pp. 552-555, 1992.

[20] W. K. Burns, R. P. Moeller, and C. Chen, "Depolarization in a single-mode optical fiber," J. Lightwave Technol., vol. 1, pp. 44-50, 1983.

[21] F. Curti, B. Daino, G. De Marchis, and F. Matera, "Statistical treatment of the evolution of the principal states of polarization in single-mode fibers," J. Lightwave Technol., vol. 8, pp. 1162-1166, 1990.

[22] C. D. Poole and D. Favin, J. Lightwave Technol., to be published.

[23] M. Bertelsmeir and W. Zschunke, "Linearization of broadband optical transmission systems by adaptive predistortion," Frequenz, vol. 38, pp. 206-212, 1984.

[24] G. N. Watson, A Treatise on the Theory of Bessel Functions. New York: Macmillan, 1948.

[25] L. J. Cimini, I. M. I. Habbab, R. K. John, and A. A. M. Saleh, "Preservation of polarization orthogonality through a linear optical system," Electron. Lett., vol. 23, pp. 1365-1366, 1987.

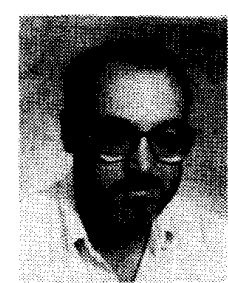

Craig D. Poole was born in Clinton, Connecticut on October 1, 1954.

$\mathrm{He}$ received the B.S. degree in physics from the Massachusetts Institute of Technology Cambridge, Massachusetts in 1977 and the M.S. and $\mathrm{Ph}$.D. degrees in electrical engineering from the University of Southern California, Los Angeles, California in 1980 and 1984 , respectively.

In 1984 he joined AT \& T Bell Laboratories, Holmdel, New Jersey, where he worked in the Undersea Cable Laboratory. In 1986 he joined the Lightwave Systems Research Department at AT \& T's Crawford Hill Laboratory, where he has engaged in research on the polarization properties of optical fiber and on fiber-based devices for lightwave systems.

Dr. Poole is a member of the Institute of Electronics and Electrical Engineers and the Optical Society of America.

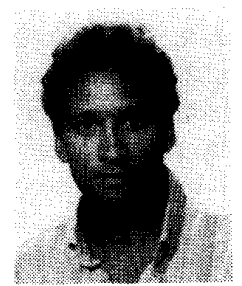

Thomas E. Darcie was born in Kitchener, Ontario, Canada, on April 1, 1956. He received the B.Sc. degree in physics from the University of Waterloo in 1977 , and graduated from the University of Toronto Institute for Aerospace Studies with the M.A.Sc. degree in 1978 and the Ph.D. degree in 1982.

In 1982 he joined the technical staff of AT \& T Bell Laboratories, at Crawford Hill, Holmdel, New Jersey, where he studied gasdynamics and particle transport in optical fiber fabrication processes. Since 1984 he has investigated the nonlinear response characteristics of semiconductor lasers, the capabilities of lightwave systems that use subcarrier multiplexing and the performance of resonant highfrequency receivers. His research on coherent lightwave techniques includes image-rejection heterodyne receivers, polarization-diversity receivers, and nonlinear interactions in semiconductor optical amplifiers. Most recently, he has studied laser and system requirements for lightwave cable television systems. He is presently head of the Lightwave Communications Research Department at Crawford Hill. 